\title{
Neutrophil cytoplasmic antibodies (p-ANCA) in ulcerative colitis
} P M Ellerbroek, M Oudkerk Pool, B U Ridwan, K M Dolman, B M E von Blomberg,
A E G Kr von dem Borne, S G M Meuwissen, R Goldschmeding
Department of Immunological Haematology, Central Laboratory of The Netherlands Red Cross Blood Transfusion Service, Amsterdam, The Netherlands P M Ellerbroek B U Ridwan $\mathrm{K} M$ Dolman A E G Kr von dem Borne R Goldschmeding

Department of Gastroenterology of the Free University Hospital, Amsterdam M Oudkerk Pool B U Ridwan $B M E$ von Blomberg S G M Meuwissen

Department of Haematology, Academic Medical Center, Amsterdam A E G Kr von dem Borne

Department of Pathology, Academic Medical Center, Amsterdam R Goldschmeding Correspondence to: Dr R Goldschmeding Department of Patholog (H2), Academic Medical Center, Meibergdreef 9 , Center, Meibergdreef 9, 1105 AZ Amster.

Accepted for publication 7 October 1993

\begin{abstract}
Aims-To study ulcerative colitis associated neutrophil cytoplasmic antibodies (p-ANCA) in respect of class and subclass distribution, antigen specificity, and (sub)cellular localisation of the antigen(s) to which these antibodies are directed.

Methods-p-ANCA positivity was determined using the standard indirect immunofluorescence test (IIFT). The immunoglobulin (Ig) subclass distribution of p-ANCA was investigated using monoclonal antibodies directed against IgG1, IgG2, IgG3, and IgG4. Intracellular antigen localisation studies were performed on (fractionated) neutrophils using antigen-specific antibodies.

Results-In contrast to vasculitis associated ANCA, ulcerative colitis p-ANCA are mainly of IgG1 and IgG3 subclass and lack IgG4. Ulcerative colitis p-ANCA are myeloid specific. IIFT data indicate that the related antigen(s) seem(s) to be located not in the cytosol, but in the granules (most likely the azurophil granules) of the neutrophil.

Conclusions-p-ANCA in ulcerative colitis have a different immunoglobulin subclass distribution than the ANCA of systemic necrotising vasculitis and necrotising and crescentic glomerulonephritis. This may point to differences in immune regulation between these diseases. Both cathepsin $G$ and lactoferrin are recognised by a subpopulation of ulcerative colitis p-ANCA. In our series, eight out of $36(22 \%)$ of ulcerative colitis associated p-ANCA react with lactoferrin and seven $(19.5 \%)$ other sera with cathepsin G. None of them recognised both antigens. The main target antigen(s) of ulcerative colitis $\mathbf{p}$-ANCA still remain(s) to be identified.
\end{abstract}

(f Clin Pathol 1994;47:257-262)

Anti-neutrophil cytoplasmic antibodies (ANCA) have proved their value in the diagnosis of vasculitic disorders, such as Wegener's granulomatosis, systemic necrotising vasculitis, and necrotising crescentic glomerulonephritis. ${ }^{1}$ In these diseases the ANCA titre correlates with disease activity. Exacerbations are preceded by a rise in the ANCA titre in the patient's serum, so these antibodies may have a pathogenetic role. ${ }^{1-3}$
The related antigens have been identified as enzymes located in the granules of neutrophils. C-ANCA in Wegener's granulomatosis are directed against proteinase 3 . p-ANCA in systemic necrotising vasculitis and necrotising crescentic glomerulonephritis are mainly directed against myeloperoxidase and rarely against neutrophil elastase. ${ }^{4-9}$

In 1983 Nielsen et al described (peri-) nuclear staining of neutrophils fixed in ethanol in the sera of patients with ulcerative colitis, using a fixed cell indirect immunofluorescence technique (IIFT). This pattern was similar to that of granulocyte specific antinuclear antibodies (GS-ANA) found in the sera of patients with rheumatoid arthritis. It has been reported in several studies that these antibodies are present in the sera of $25 \%$ of patients with ulcerative colitis and only in 3\% of those with Crohn's disease. ${ }^{1011}$ Saxon et al reported similar perinuclear staining of neutrophils, now called p-ANCA, in the sera from patients with inflammatory bowel disease. In their study $68 \%(n=25)$ of the ulcerative colitis sera and $12 \%(n=25)$ of the Crohn's disease sera reacted positively in the IIFT. ${ }^{12}$ We recently screened 317 sera from patients with inflammatory bowel disease and 49 control sera using the IIFT; $79 \%$ of the ulcerative colitis sera, $13 \%$ of the Crohn's disease sera, and $9 \%$ of the control sera showed a positive reaction with neutrophilic granulocytes. p-ANCA titres were not correlated in ulcerative colitis or in Crohn's disease with disease activity, duration of illness, localisation, extent of disease, previous bowel operations or medical treatment. The clinical importance of $\mathrm{p}$-ANCA positive and negative subsets in both groups of patients thus remains unexplained. ${ }^{13}$ To obtain a better insight into the background and importance of this autoimmune phenomenon in ulcerative colitis, we therefore investigated the size, class and the subclass distribution of ulcerative colitis related p-ANCA immunoglobulins as well as the subcellular localisation of the antigen(s) to which these antibodies are directed.

\section{Methods}

Sera from 36 patients with ulcerative colitis, positive for IgG p-ANCA staining in the IIFT on ethanol-fixed granulocytes, were selected for further study. The presence of antinuclear antibodies in the sera was excluded in a routine assay using rat liver as a substrate. Ulcerative colitis was diagnosed on the basis of clinical, radiological, histological, and 
endoscopic evidence. ${ }^{14}$ Sera containing antibodies against myeloperoxidase, proteinase 3, elastase, lactoferrin, and cathepsin G, as detected by capture ELISA, served as controls in all the assays used in this study. Sera negative in the IIFT were obtained from patients with Crohn's disease and from healthy blood donors to serve as controls.

BLOOD CELLS AND LEUKAEMIA CELL LINES Granulocytes and lymphocytes were obtained by Ficoll sedimentation of EDTA anticoagulated buffy coats of regular blood donations from healthy donors. Leucocytes were also isolated from blood of a patient with chronic myeloid leukaemia (CML). Lymphocytes were aspirated from the interface and washed in $0.2 \%$ bovine serum albumin-phosphate buffered saline (BSA-PBS). Erythrocytes were lysed by ammonium chloride and the remaining granulocyte pellet was washed three times with PBS. Thrombocytes were isolated by centrifuging EDTA anticoagulated blood at $400 \times \mathrm{g}$, followed by centrifugation of the platelet rich plasma at $1500 \times \mathrm{g}$. The pelleted platelets were suspended in EDTA-PBS buffer. Monocytes were isolated by successive isopycnic centrifugation and counterflow elution, as described by Roos et al. ${ }^{15}$ The histiocytic cell line U937 was cultured in RPMI 1640 medium (Gibco, Paisley, Scotland).

PREPARATION OF CYTOPLASTS AND

KARYOPLASTS

Granulocytes from a healthy donor were fractionated by discontinuous Ficoll gradient centrifugation with cytochalasin $\mathrm{B}$ (at $37^{\circ} \mathrm{C}$ ). After washing in PBS the cytoplasts (containing two thirds of the cytosol surrounded by plasma membrane) and karyoplasts (the granules, nuclei and one third of the cytosol surrounded by plasma membrane) were either cytospinned and fixed in ethanol for IIFT, or coated as a monolayer to microtitre plates in Hanks's buffered salt solution and fixed with $100 \%$ methanol for enzyme linked immunosorbent assay (ELISA). ${ }^{16}$

\section{PREPARATION OF NEUTROPHIL GRANULE \\ PROTEIN EXTRACT}

Isolated neutrophils were disrupted by cavitation in a nitrogen bomb. ${ }^{17}$ Nuclei and unbroken cells were pelleted by centrifugation at $500 \times g$ for 10 minutes (at $4^{\circ} \mathrm{C}$ ). The supernatant fluid was centrifuged at $35000 \times g$ for 20 minutes (at $4^{\circ} \mathrm{C}$ ). The pelleted mixed granules were resuspended in PBS- $0.1 \%(\mathrm{v} / \mathrm{v})$ Triton X-100 and sonicated at $45 \mathrm{kHz}$ on melting ice for three periods of 10 seconds each. Thereafter, soluble and insoluble material were separated by centrifugation at $220000 \times g$ for 1 hour (at $4^{\circ} \mathrm{C}$ ). The supernatant "extract" was dialysed against PBS. ${ }^{17}$

IIFT

The standard IIFT was performed according to Wiik's method. ${ }^{18}$ Isolated human blood cells were either cytospinned on slides (Menzel Gläser, Omnilabo International, Breda, The Netherlands) or smeared on eight-well Nutacon slides (Nutacon, Schiphol, The Netherlands) and fixed in $96 \%$ ethanol for 15 minutes (at $4^{\circ} \mathrm{C}$ ). Thereafter, the slides were incubated with serum diluted 1 in 16 in 4\% BSA-PBS for 1 hour, followed by washing in PBS. Bound antibodies were detected by fluorescein isothiocyanate (FITC)-labelled F(ab')2 fragments of rabbit anti-human IgG. The slides were examined by fluorescence microscopy. In some experiments the smears were fixed with phosphate buffered formalin and acetone solution, $\mathrm{pH} 6 \cdot 8$, containing $9 \cdot 25 \%(\mathrm{v} / \mathrm{v})$ formalin and $45 \%(\mathrm{v} / \mathrm{v})$ acetone for 2 seconds at $4^{\circ} \mathrm{C}$. The immunoglobulin subclass distribution of p-ANCA in ulcerative colitis was investigated using monoclonal antibodies directed against IgG1, IgG2, IgG3, and IgG4, followed by FITC-conjugated goat anti-mouse immunoglobulin. For the detection of IgM and IgA binding, FITC-labelled F(ab') 2 fragments of rabbit anti-human IgM or IgA were used. The optimal dilutions were determined by titration on positive and negative control sera.

To examine the reactivity of sera with the plasma membrane, granulocytes were fixed in suspension with $1 \%$ paraformaldehyde for 5 minutes (at $4^{\circ} \mathrm{C}$ ). Bound antibodies were detected by fluorescein-conjugated $F\left(a b^{\prime}\right) 2$ fragments of sheep anti-human immunoglobulin and measured by fluorescence flow cytometry. ${ }^{19}$

\section{CAPTURE ELISA}

This assay is described in detail in reference 5. In brief, goat anti-mouse IgG antibodies (Jackson Immuno Research Laboratories Inc, Avondale, Philadelphia, USA) were coated on microtitre plates (Maxisorp, Nunc). After incubation with monoclonal antibodies and washing, the plates were incubated with granule extract to allow the monoclonal antibodies to capture their respective antigens from a neutrophil granule extract. After another wash patient serum was added. Bound patient antibodies were detected by affinity purified alkaline phosphatase conjugated goat anti-human IgG. ${ }^{5}$

Ficoll, protein A-sepharose CL-4B, and sephacryl S-400 were obtained from Pharmacia Fine Chemicals (Uppsala, Sweden). Paraformaldehyde, formalin, and methanol were purchased from Merck (Darmstadt, Germany). Acetone was from OPG Farma (Utrecht, The Netherlands). Ethanol was from Brocacef (Maarssen, The Netherlands), and EDTA from Aldrich Chemie (Brussels, Belgium). Cytochalasin B, Triton X-100, and Sigma 104 alkaline phosphatase substrate were from Sigma (St Louis, Missouri, USA). Bovine serum albumin was obtained from Boseral (Boxtel, The Netherlands). ACA 44 was from IBF (Villeneuve la Garenne, France).

\section{ANTISERA}

FITC-labelled $F\left(a^{\prime}\right) 2$ fragments of rabbit anti-human IgG were obtained from Dakopatts D/A (Denmark). Alkaline phosphatase labelled goat anti-human IgG/goat 
Figure 1 IgG, IgM, and IgA class titres of ulcerative colitis related $p-A N C A s$, anti-proteinase $3 A N C A$, and anti-myeloperoxidase ANCAs.

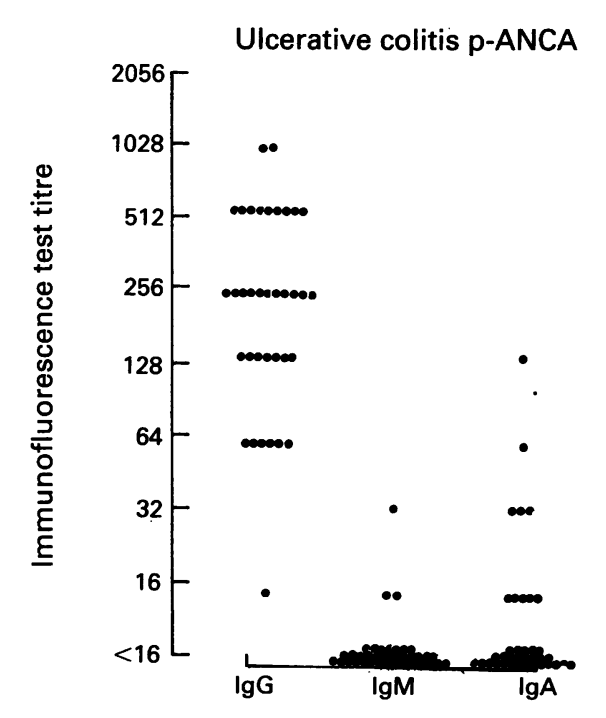

Ig class distribution
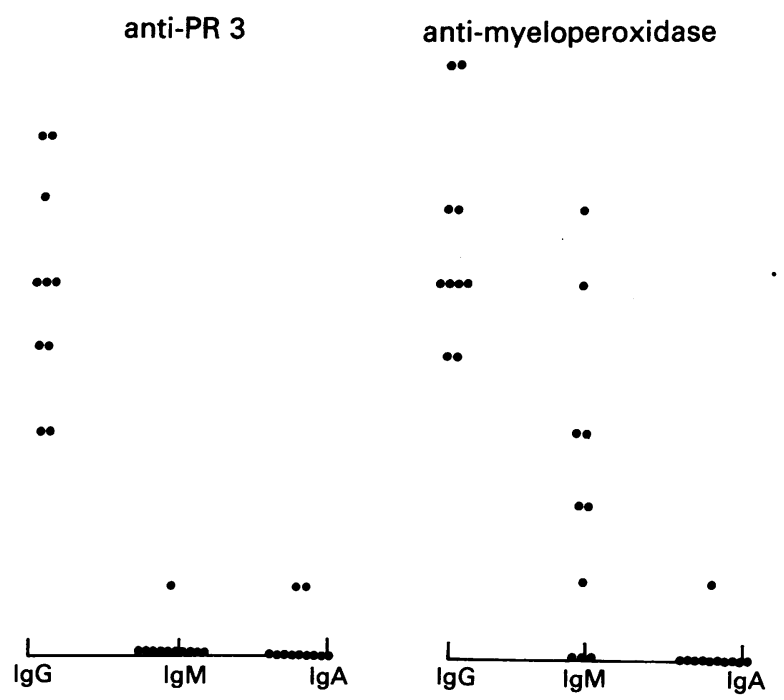

anti-mouse IgG and unlabelled goat antimouse IgG were obtained from Jackson Immuno Research Laboratories Inc. FITCcoupled $F\left(a b^{\prime}\right) 2$ fragments of sheep antihuman IgG (S26H17F), FITC-coupled goat anti-mouse IgG, goat anti-human IgG, rabbit anti-human $\operatorname{IgM}$, peroxidase bound rabbit anti-human IgM, FITC-coupled rabbit antihuman IgA (KH26H14), and FITC-coupled rabbit anti-human $\operatorname{IgM}(\mathrm{K} 26 \mathrm{H} 15)$ were produced at the Central Laboratory of the Netherlands Red Cross Blood Transfusion Service (Amsterdam) as were the monoclonal antibodies anti-human IgG1 (clone 161.2M), anti-human IgG2 (clone 162.1M), anti-human IgG3 (clone 163.1M), anti-human IgG4 (clone $164.4 \mathrm{M}$ ), and 12.8 (IgG1 anti-proteinase 3 ), 4.15 (IgG1 anti-myeloperoxidase), 14.15 (IgG1 anti-neutrophil lactoferrin), and AME-1 (IgG1 anti-glycophorin A). AHN-11 (IgG1 anti-cathepsin G) was a gift from Dr KM Skubitz (University of Minnesota Medical School, Minneapolis, USA). NP-57 (IgG1 anti-neutrophil elastase) was a gift from $\mathrm{Dr}$ DY Mason (John Radcliffe Hospital, Oxford, England).

\section{Results}

IgG CLASS AND SUBCLASS DISTRIBUTION OF ULCERATIVE COLITIS ASSOCIATED p-ANCA In most ulcerative colitis p-ANCA sera IgG class antibodies only were detected. Additional IgM p-ANCA binding was found in three out of 36 sera and IgA p-ANCA in 10 out of 36 sera. The occurrence of IgM and IgA class ANCA was also tested in 20 control sera, either positive for IgG class ANCA directed against proteinase $3(n=10)$ or $I g G$ p-ANCA against myeloperoxidase $(n=10)$ (fig 1). Anti-proteinase 3 , as well as antimyeloperoxidase p-ANCA, were also mainly IgG class, but in six out of 10 sera with IgG antibodies against myeloperoxidase there was a strong IgM class p-ANCA as well. These sera did not contain rheumatoid factor, as determined by the latex fixation test.

Ulcerative colitis p-ANCA IgG was mainly of IgG1 and IgG3 subclasses. No IgG4 was detected (fig $2 \mathrm{~A}$ ). In contrast, vasculitisassociated anti-proteinase 3 ANCA and antimyeloperoxidase ANCA IgG contained relatively high concentrations of specific IgG4 (fig $2 \mathrm{~B}$ and $\mathrm{C}$ ).

\section{FIXATION STUDIES}

To study the effects of different fixation procedures on the staining patterns obtained in the IIFT, 20 ulcerative colitis p-ANCA sera were tested both on cytospins of granulocytes treated with ethanol (standard IIFT) and on slides treated with phosphate buffered formalin-acetone solution. On formalin-acetone fixed cells, three out of 20 ulcerative colitis p-ANCA sera were negative; three other sera also showed vague cytoplasmic staining. The remaining (14/20) sera showed a granular cytoplasmic staining pattern, although weaker in intensity than that on ethanol fixed cells (not shown).

\section{CELL SPECIFICITY}

All ulcerative colitis p-ANCA positive sera were also assayed for reactivity with cytospin slides of thrombocytes, lymphocytes, and eosinophilic granulocytes. None of these cells was stained by ulcerative colitis p-ANCA sera. When tested on monocytes, however, 21 sera showed p-ANCA reactivity with $10-40 \%$ of the monocytes. Thirteen p-ANCA positive sera also reacted positively with some promonocytic U937 cell line cells. Thus ulcerative colitis associated p-ANCA are myeloid specific and a $100 \%$ reaction is found only with neutrophilic granulocytes (table).

\section{REACTIVITY WITH ISOLATED MYELOID} GRANULE PROTEINS

In contrast to control c-ANCA and p-ANCA positive sera from patients with Wegener's granulomatosis and other vasculitides, ulcerative colitis p-ANCA sera did not react with captured proteinase 3, myeloperoxidase, or elastase. A few of the ulcerative colitis sera did recognise lactoferrin or cathepsin G. Eight of 36 sera bound to lactoferrin. Seven other sera 


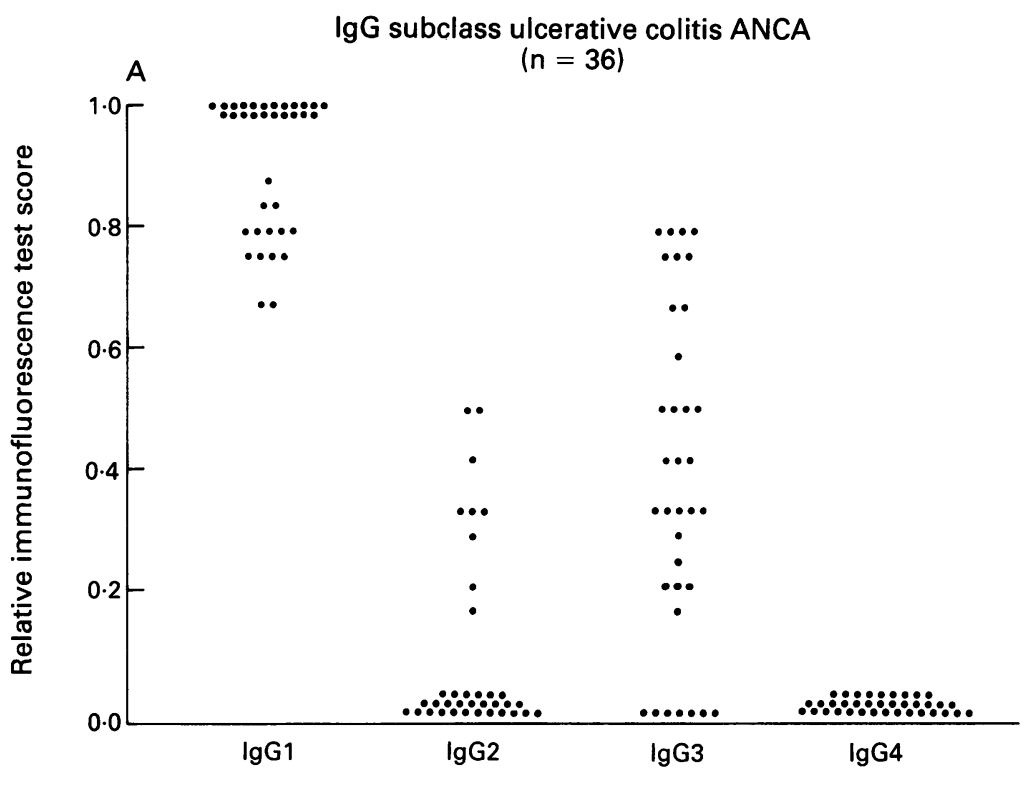

lgG subclass anti-proteinase ANCA

$$
(n=10)
$$

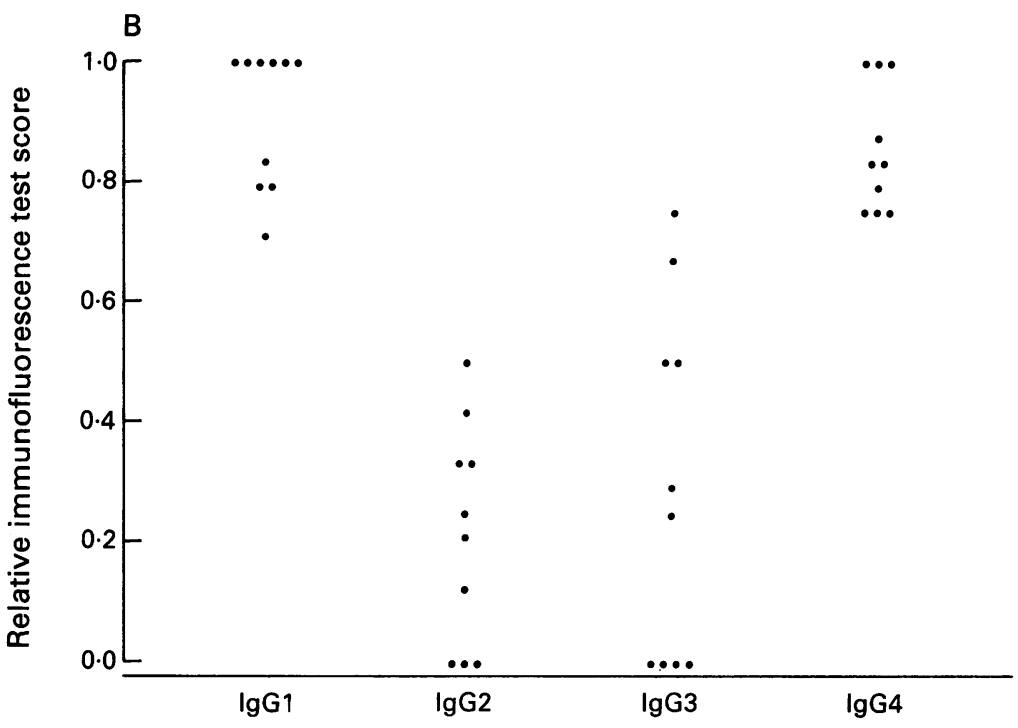

IgG subclass anti-myeloperoxidase ANCA $(n=10)$

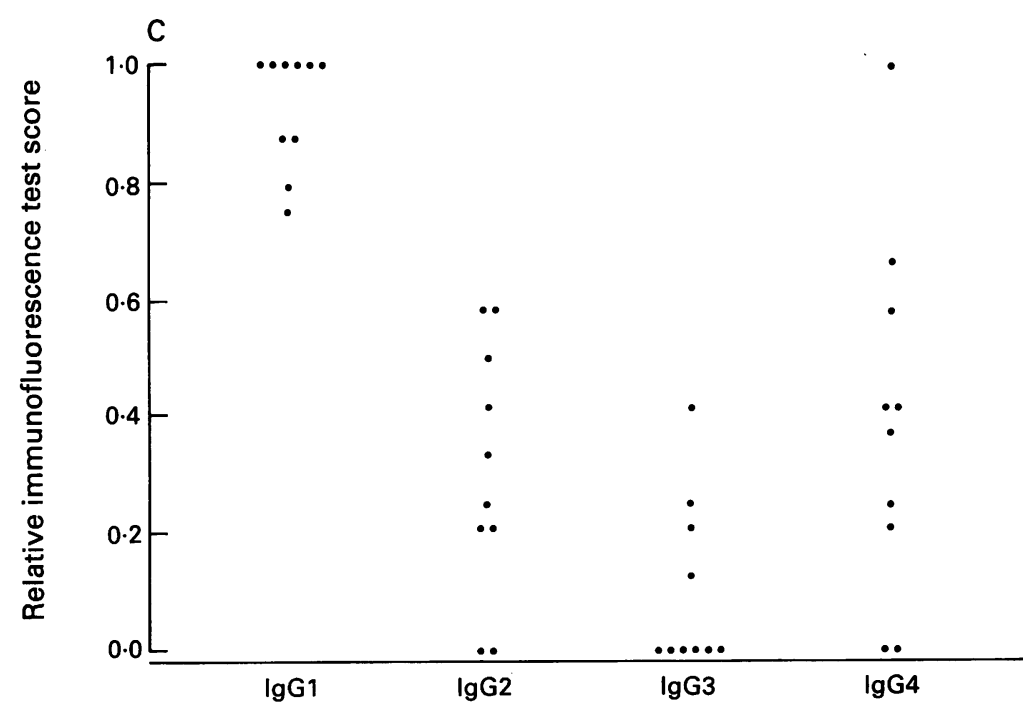

Figure 2 (A) IgG subclass distribution of p-ANCA; (B) anti-proteinase 3 ANCAs; (C) anti-myeloperoxidase ANCAs as detected by IIFT. To enable comparison of results with sera with different (total) IgG ANCA titres for each serum, the IgG subclass titre is expressed relative to that obtained with FITC coupled goat anti-human IgG (total).
Reactivity of $p-A N C A$ positive ulcerative colitis sera with different cell types in IIFT

\begin{tabular}{lcc}
\hline Cell type & $\begin{array}{l}\text { Positive sera } \\
\text { (total } n=36)\end{array}$ & $\begin{array}{l}\text { Percentage of } \\
\text { cells stained }\end{array}$ \\
\hline Neutrophils & 36 & 100 \\
Eosinophils & 0 & 0 \\
Monocytes & 21 & $10-40$ \\
Lymphocytes & 0 & 0 \\
Thrombocytes & 0 & 0 \\
U937 & 13 & $10-40$ \\
\hline
\end{tabular}

bound to cathepsin $G$ in a capture ELISA using granule extract from chronic myeloid leukaemia cells (fig 3 ). Three of these seven sera showed only marginally increased binding.

\section{SUBCELLULAR LOCALISATION OF P-ANCA} ASSOCIATED ANTIGEN(S)

Using immunofluorescence on paraformaldehyde-fixed neutrophils in suspension (IIFT), none of the ulcerative colitis p-ANCA sera reacted positively with the granulocyte plasma membrane. Using IIFT on cytospins as well as a solid phase ELISA, none of 10 ulcerative colitis p-ANCA sera tested reacted with the cytoplasts of neutrophils, but all 10 sera reacted positively with the karyoplast fraction of the same cells. Therefore, the antigens recognised by ulcerative colitis p-ANCA do not seem to be located in the cytosol or plasma membrane, but in cellular structures with higher sedimentation rates-the granules, for example.

In additional experiments using solid-phase ELISAs 11 out of 36 ulcerative colitis pANCA sera reacted with a crude preparation of $\left(\mathrm{N}_{2}\right.$-cavitated) neutrophil granule protein extract from which the nuclei and cytosol had been removed by differential centrifugation. The same 11 sera reacted positively with a comparable preparation extracted from CML cells. Five other sera reacted positively only with the CML preparation but not with that from mature neutrophils. Reactivity with the various preparations in these ELISAs was not related to the titre of the sera in the IIFT. Western blotting and radioimmunoprecipitation did not identify bands specifically recognised by ulcerative colitis p-ANCA sera (data not shown).

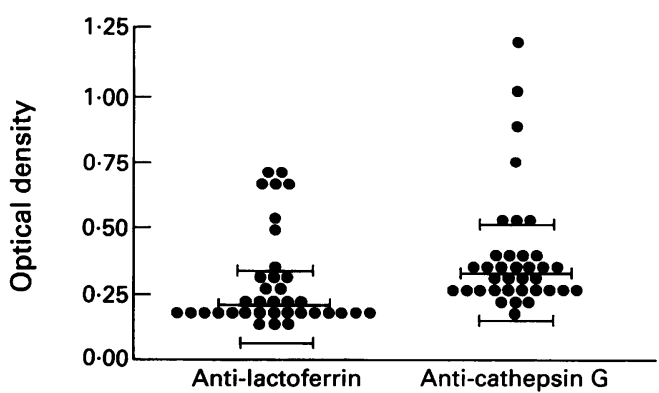

Figure 3 Capture ELISA for detection of antibodies against lactoferrin and cathepsin $G$. Thirty six $p-A N C A$ positive ulcerative colitis sera were tested. None of the sera recognised both antigens. Lines indicate means $\pm 2 S D$ of 10 normal control sera included in this experiment. Normal range is representative as evidenced by separate experiments including larger numbers of controls (not shown). 


\section{Discussion}

We investigated the nature of the neutrophil reactive immunoglobulin(s) in ulcerative colitis sera that produce $\mathrm{p}$-ANCA staining in the IIFT on ethanol fixed neutrophil slides. pANCA in ulcerative colitis sera, like many other autoantibody sera, are mainly IgG class antibodies of the IgG1 and the IgG3 subclass and lack IgG4 antibodies. In this respect, however, they differ from the vasculitis associated ANCAs which have relatively high IgG4 titres. This suggests that there may be differences in the regulation of the autoimmune response in ulcerative colitis compared with the vasculitides..$^{22} 23$

Like systemic necrotising vasculitis and necrotising and crescentic glomerulonephritis associated ANCAs, p-ANCA in ulcerative colitis seem to be myeloid specific. ${ }^{1}$ In the IIFT ulcerative colitis p-ANCA reacted with both neutrophils and monocytes, although generally only a minority of monocytes became positive (table). This differential reactivity with monocyte(-subpopulation)s might be related to differences in antigen contents between immature and mature monocytes. ${ }^{24}$ The fact that, in contrast to our observations, Duerr et al found no reactivity of ulcerative colitis sera in a fixed monocyte ELISA might be explained by lower sensitivity of this test for identification of antibodies reacting with only subpopulations of these cells. ${ }^{25}$ It is possible that the ulcerative colitis associated GS-ANA, originally described by Nielsen in the early eighties are not nuclear but in fact perinuclear staining patterns, that are now called p-ANCA. ${ }^{1011}$ This p-ANCA pattern, however, is not a reliable indication of the subcellular location of the antigen(s) involved. It may be artefactually generated by the fixation process of neutrophils. The ulcerative colitis p-ANCA staining pattern in the IIFT depended on the fixation procedure, as has been described earlier for vasculitis associated p-ANCA directed against myeloperoxidase and elastase. ${ }^{26}$ For ulcerative colitis p-ANCA, we found a perinuclear staining pattern on ethanol fixed cytospins or smears; after formalin-acetone fixation the pattern was cytoplasmic. This suggests that, similar to the target antigens of vasculitis-related ANCA, the ulcerative colitis p-ANCA antigen(s) may also be located in the granules, rather than in the nucleus of neutrophils. ${ }^{27}$ The results of cell line studies, cytoplast/karyoplast separation and granule extract analysis would be compatible with localisation of the target antigen(s) of ulcerative colitis p-ANCA in the azurophilic granules of neutrophils, like the target antigens of systemic necrotising vasculitis and necrotising and crescentic glomerulonephritis ANCA. ${ }^{5-7}$ Only a minority of p-ANCA positive ulcerative colitis sera, however, gave a positive result in solid phase ELISAs on neutrophil and CML granule extracts. No specific bands could be identified by western blotting or radioimmunoprecipitation. In our routine capture ELISA for detection of vasculitis and glomerulonephritis related ANCA against proteinase 3, myeloperoxidase, and elastase, all ulcerative colitis p-ANCA sera were negative. In addition, in a solid phase ELISA on directly coated antigens (purified proteinase 3 , myeloperoxidase, elastase) no clear positive reactions were obtained. Ulcerative colitis $\mathrm{p}$ ANCA, therefore, do not seem to be directed against the same antigens as systemic necrotising vasculitis and necrotising and crescentic glomerulonephritis ANCA.

Thompson and Lee reported the detection of antibodies against lactoferrin in a patient with Wegener's granulomatosis but these antibodies do not seem to be common in this disease. ${ }^{28} \mathrm{~A}$ recent study suggests that lactoferrin antibodies may be an important target antigen of p-ANCA in patients with ulcerative colitis who also have primary sclerosing cholangitis as IgG lactoferrin antibodies were found in $50 \%$ of the sera from such patients. ${ }^{29}$ Halbwachs-Mecarelli et al reported p-ANCA activity against cathepsin $G$ in a substantial proportion of $\mathrm{p}$-ANCA positive sera in a small group of ulcerative colitis patients. ${ }^{30}$

Although we found smaller percentages of positive sera, the results of our study confirm both antigen specificities. In eight out of $36 \mathrm{p}$ ANCA positive ulcerative colitis sera we detected antibodies against lactoferrin; seven other sera contained antibodies against cathepsin G. The remaining 21 ulcerative colitis p-ANCA sera failed to show such reactivity. Efforts to characterise other antigens involved in ulcerative colitis p-ANCA reactivity, by Percoll density gradient centrifugation and immunoprecipitation, which have been successfully used in the identification of the target antigens of systemic necrotising vasculitis and necrotising and crescentic glomerulonephritis ANCA, ${ }^{5}$ have thus far not revealed their identity. Theoretically, this might also relate to the possibility of p-ANCA staining being produced by adherence of "sticky" immune complexes or aggregates rather than by antigen specific immunoglobulin binding. Therefore, size distribution of ulcerative colitis p-ANCA reactivity was studied using ulcerative colitis p-ANCA serum lacking IgM or IgA class p-ANCA after isokinetic sucrose gradient centrifugation. ${ }^{31}$ p-ANCA reactivity was found within the monomeric IgG peak (not shown). In addition, gel filtration at acid pH (performed by Wiik et al to dissociate immune complexes in GS-ANA sera from patients with rheumatoid arthritis ${ }^{20}$ ) did not reveal p-ANCA reactivity in immune complexes (not shown). Together with the generally weak and variable reactivity of the ulcerative colitis p-ANCA sera in the various assays used, this might suggest that ulcerative colitis p-ANCA are monomeric but low affinity antibodies, which may be generated in the course of chronic inflammation. Because production of IgG4 antibodies is related to prolonged antigen specific $T$ cell dependent immune stimulation, the lack of IgG4 in ulcerative colitis p-ANCA would also seem compatible with a relatively low affinity of these antibodies. Our own observation that p-ANCA positivity does not correlate with activity, extent, or duration of ulcerative colitis 
may seem to argue against chronic inflammation as a factor in the generation of ulcerative colitis p-ANCA. ${ }^{13}$ It should be realised, however, that ulcerative colitis is by definition a disease of chronic inflammation and that the formation of p-ANCA in only a subgroup of ulcerative colitis patients may depend on differences in genetic background. ${ }^{32}$

In conclusion, unlike the ANCA of systemic necrotising vasculitis and necrotising and crescentic glomerulonephritis, which are predominantly of the IgG1 and IgG4 subclasses, p-ANCA in ulcerative colitis are mainly of IgG1 and IgG3 subclasses and lack IgG4, which may point to differences in immune regulation between these groups of patients. We have clearly shown that both cathepsin $\mathrm{G}$ and lactoferrin are recognised by a minority of ulcerative colitis p-ANCA sera, but none of them are recognised both antigens. Finally, we have excluded the possibility that these antibodies are artefacts related to circulating immune complexes in these patients.

We thank Professor AS Peña for helpful comments.

1 Gross WL, Schmitt WH, Csernok E. ANCA and associated diseases: immunodiagnostic and pathogenetic aspects. Clin Exp Immunol 1993;91:1-12.

2 Falk RJ, Terrell RS, Charles LA, Jennette JC. Anti-neutrophil cytoplasmic autoantibodies induce neutrophils to rophil cytoplasmic autoantibodies induce neutrophils to degranulate and produce oxygen radical

3 Kallenberg CGM, Cohen Tervaert JW, van der Woude FJ, Goldschmeding $R$, von dem Borne AEG, Weening JJ. Viewpoint autoimmunity to lysosomal enzymes: new clues to vasculitis and glomerulonephritis? Immunology Today 1991;61:61-4

4 Kao RC, Wehner NG, Skubitz KM, Gray BH, Hoidal JR. Proteinase 3, a distinct human polymorphonuclear leukocyte proteinase that produces emphysema in hamsters. $¥$ Clin Invest 1989;82:1963-73.

5 Goldschmeding $R$, van der Schoot $C E$, ten Bokkel Huinink $\mathrm{D}$, et al. Wegener's granulomatosis autoantibodies identify a novel diisopropyl-fluorophosphatebodies identify a novel diisopropyl-fluorophosphatebinding protein in the lysosomes of norm

6 Lüdemann J, Utecht B, Gross WL. Anti-neutrophil cytoplasm antibodies in Wegener's granulomatosis recognize an elastinolytic enzyme. $\mathcal{F}$ Exp Med 1990;171:357-62.

7 Savige JA, Gallicchio M, Georgiou T, Davies DJ. Diverse target antigens recognized by circulating antibodies in anti-neutrophil cytoplasm antibody-associated renal vasculitides. Clin Exp Immunol 1990;82:238-43.

8 Falk RJ, Hogan SL, Wilkman RS, et al. Myeloperoxidase specific anti-neutrophil cytoplasmic autoantibodies (myeloperoxidase-ANCA). Neth $¥$ Med 1990;36:121-5.

9 Lee SS, Adu D, Thompson RA. Anti-myeloperoxidase antibodies in systemic vasculitis. Clin Exp Immunol 1990;79:41-6.

10 Nielsen H, Wiik A, Elmgreen J. Granulocyte-specific antinuclear antibodies in ulcerative colitis. APMIS 1983; 91:23-6.

11 Wiik A. Granulocyte-specific antinuclear antibodies. Allergy 1980;35:263-89.
12 Saxon A, Shanahan F, Landers C, Ganz T, Targan S. A distinct subset of antineutrophil cytoplasmic antibodies is associated with inflammatory bowel disease. $\mathcal{F}$ Allergy Clin Immunol 1990;86:202-10.

13 Oudkerk Pool M, Ellerbroek PM, Ridwan BU, et al. Serum antineutrophil cytoplasmic autoantibodies in inflammatory bowel disease are mainly associated with ulcerative colitis. A correlation study between perinuulcerative colitis. A correlation study between perinu-
clear antineutrophil cytoplasmic autoantibodies and clear antineutrophil cytoplasmic autoantibodies and
clinical parameters, medical, and surgical treatment. Gut clinical parameters

14 Lennard-Jones JE. Classification of inflammatory bowel disease. Scand $\mathcal{F}$ Gastroenterol 1989;24(suppl 170):2-6.

15 Roos D, de Boer M. Purification and cryopreservation of phagocytes from human blood. Methods in Enzymology 1986:225-43.

16 Roos D, Voetman AA, Meerhof LJ. Functional activity of enucleated human polymorphonuclear leukocytes. $\mathcal{f}$ Cell Biol 1983;97:368-77.

17 Borregaard N, Heiple JM, Simons ER, Clark RA. Subcellular localization of the b-Cytochrome component of the human neutrophil microbial oxidase: translonent of the human neutrophil microbial oxidase: tra
cation during activation. $\Im$ Cell Biol 1983;97:52-61.

18 Wiik A. Delineation of a standard procedure for indirect immunofluorescence detection of ANCA. APMIS 1989; 97:12-15.

19 Verheugt FWA, von dem Borne AEG, Decary F, Engelfriet CP. Detection of granulocyte alloantibodies by an indirect immuno-fluorescence test. $\mathrm{Br} \mathcal{F} \mathrm{Haematol}$ 1977;36:533.

20 Wiik A. Circulating immune complexes involving granulocyte-specific antinuclear factors in Felty's syndrome and rheumatoid arthritis. APMIS 1975;83:354-64.

21 Slaper Cortenbach ICM, Admiraal LG, Kerr JM, van Leeuwen EF, von dem Borne AEG, Tetteroo PAT. Flow-cytometric detection of terminal deoxynucleotidyl Flow-cytometric detection of terminal deoxynucleotidyl transferase and other intracellular antigens in combina-
tion with membrane antigens in acute lymphatic tion with membrane antigens in acute lymphatic
leukemias. Blood 1988;72:1639-44.

22 Schur PH. IgG subclasses-a review. Ann Allergy 1987; 58:89-96.

23 E Brouwer, Cohen Tervaert JW, Horst G, et al. Predominance of IgG1 and IgG4 subclasses of anti-neutrophil cytoplasmic autoantibodies (ANCA) in patients with Wegener's granulomatosis and clinically related disorders. Clin Exp Immunol 1991;83:379-86.

24 Campbell EJ, Cury JD, Shapiro SD, Goldberg GI, Welgus HG. Neutral proteinases of human mononuclear phagocytes: Cellular differentiation markedly alters cell phenocytes: Cellular differentiation markedly alters cell phenotype for serine proteinases, metaloproteases, and tissue
inhibitor of metalloproteases. $\mathcal{F}$ Immunol 1991;146: inhibitor

25 Duerr RH, Targan SR, Landers CJ, et al. Neutrophil cytoplasmic antibodies: a link between primary sclerosing cholangitis and ulcerative colitis. Gastroenterology 1991; 100:1385-91.

26 Falk RJ, Jennette JC. Anti-neutrophil cytoplasmic autoantibodies with specificity for myeloperoxidase in patients with systemic vasculitis and idiopathic necrotizing and crescentic glomerulonephritis. $N$ Engl f Med 1988;318: 1651-7.

27 Snook JA, Chapman RW, Fleming K, Jewell DP. Antineutrophil nuclear antibody in ulcerative colitis, Crohn's neutrophil nuclear antibody in ulcerative colitis, Crohn's
disease and primary sclerosing cholangitis. Clin Exp disease and primary

28 Thompson RA, Lee SS. Antineutrophil cytoplasmic antibodies. Lancet 1989;i:670-1.

29 Peen E, Almer S, Bodemar G, et al. Anti-lactoferrin antibodies and other types of ANCA in ulcerative colitis, primary sclerosing cholangitis, and Crohn's disease. Gut 1993;34:56-62.

30 Halbwachs-Mecarelli L, Nusbaum P, Noel LH, et al. Antineutrophil cytoplasmic antibodies (ANCA) directed against cathepsin $\mathrm{G}$ in ulcerative colitis, Crohn's disease, and primary sclerosing cholangitis. Clin Exp Immunol and primary scler

31 Noll $\mathrm{H}$. Characterization of macromolecules by constant velocity sedimentation. Nature 1967;215:360-3.

32 Yang $\mathrm{H}$, Rotter II, Toyoda $\mathrm{H}$, et al. Ulcerative colitis: a genetic heterogenous group defined with genetic (DR2) and subclinical (ANCAs) markers. Gastroenterology 1992;102:A716. 